\title{
Design and Simulation of a Wideband Hybrid Envelope Tracking Power Amplifier Using LDMOS Transistor
}

\author{
Iman Arianyan, Abdolali Abdipour, Abbas Mohammadi, and Ehsan Arianyan
}

\begin{abstract}
There are many methods for improving the efficiency of the power amplifier, one of which is Hybrid EER that we use. In this technique, the signal is divided into two parts. In a path the information in the envelope of the signal is detected and is applied to the drain of the transistor. By this method the voltage of the drain is variable and is related to the amplitude of the input signal. In another path the signal is fed to the RF amplifier. In this paper we introduce EER, ET and HEER techniques and explain the envelope amplifier. Design of the envelope amplifier is very important in the system; since it plays a chief role in the total efficiency. We have designed the power amplifier using the MRF6S27015N MOTOROLA transistor in LDMOS technology and applied HEER to our amplifier. It caused more than $50 \%$ of $\mathrm{PAE}$ in a wide range of input power.
\end{abstract}

Index Terms-Envelope elimination and restoration, envelope amplifier, power amplifier.

\section{INTRODUCTION}

Two main characteristic of a communication system is high linearity and high efficiency. Power amplifier is the most important part in a communication system. The reason is that the power amplifier uses the most amount of energy in the system and its good efficiency can decrease the amount of heat generated, and therefore boost the performance of the total system. On a communication satellite system, up to $50 \%$ of the total power can be used by the power amplifier (PA) in the transmitter [1]. Moreover, more efficiency may result in less energy consumption and reduce the cost of the system. Modern communication system needs high data rates. Consequently, they prefer non constant modulation techniques, such as QAM, which may have large peak to average ratio. We may use back off to maintain linearity of the power amplifier but this may result in decreasing the efficiency.

Some techniques are proposed to overcome this problem. As a good candidate for the techniques, LINC, $\Delta \Sigma$ modulation, Doherty, and EER/Polar modulator have been suggested. The first two techniques show a high linearity with a moderate efficiency [2], [3] and the latter two techniques show a high efficiency with a moderate linearity characteristic generally [4], [5]. However, applying the digital predistortion (DPD) technique to the latter two

Manuscript received September 13, 2012; revised October 24, 2012.

The authors are with the Department of Electrical Engineering Department, Amirkabir University of Technology, Tehran, Iran (e-mail: iman_aryanian@aut.ac.ir, abdipour@aut.ac.ir, abm125@aut.ac.ir, ehsan_arianyan@aut.ac.ir) techniques can enhance the moderate linearity adequately.

\section{A. Paper Organization}

In the sections that follow, we first review envelope tracking, Envelope elimination and restoration, and hybrid EER techniques, and then explain envelope amplifier. After that, we talk about designing the RF amplifier, and how we applied envelope tracking to increase the power added efficiency and the results are explained.

\section{ENVELOPE TRACKING}

Fig. 1 shows the block diagram of traditional ET systems. ET uses a linear PA and a controlled supply voltage, which tracks the input envelope. When the supply voltage tracks the instantaneous envelope modulation signal, it is called Wide Bandwidth ET (WBET) [6]-[9]; when the supply voltage tracks the long-term average of the input envelope power, it is called Average ET (AET) [10]-[11]; when the supply voltage switches to different step levels according to the input envelope power, it is called Step ET (SET) [12], [13]. In ET we dynamically bias the RF transistor so that the RF transistor working in the high efficiency compression region over a wide dynamic range of output power and improve the $\mathrm{PA}$ average efficiency.

\section{ENVELOPE ELIMINATION AND RESTORATION}

EER uses a combination of a high efficiency switch-mode PA with an envelope re-modulation circuit [14]. Fig. 2 shows the block diagram of traditional EER systems. First, the RF signal is exerted to the limiter; the result is the phase modulated signal and is inputted to the switch mode PA. In another path the envelope signal is detected and is remodulated to the signal through the drain or the collector of the RF transistor. In theory, since the RF transistor is always operating in a switching mode, EER is more efficient than ET. However, the PA for a conventional EER transmitter has been designed to achieve peak efficiency at the peak drain voltage region and does not have a good efficiency at the average region. It causes a degradation of the overall efficiency of the EER transmitter [5].

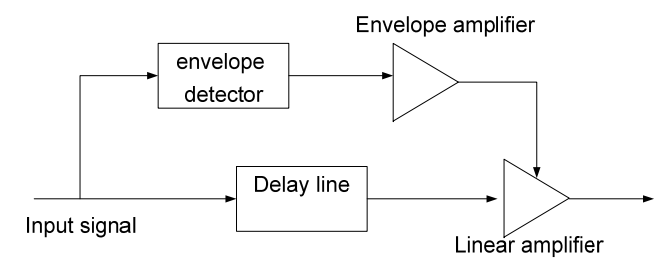

Fig. 1. Envelope tracking block diagram 


\section{HYBRID EER}

To employ the high efficiency operation of EER and at thesame time reduce the strict necessities of bandwidth and time-alignment, the "hybrid" EER structure was proposed recently [15]. Fig. 3 shows the block diagram of the hybrid EER system, where the PA input signal is a complex-modulated signal with variable envelope like ET, but the difference is that the PA is designed to operate in the switching-mode at higher input powers. The hybrid EER has some benefits compared with traditional EER systems. It has lower RF bandwidth and envelope bandwidth requirement because its input signal is not limited. Moreover, it can provide higher gain and therefore higher average PAE and finally, it has lower sensitivity to the time-mismatch between envelope and RF path.

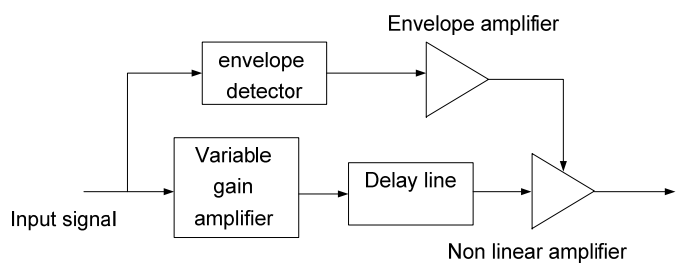

Fig. 2. Envelope elimination and restoration block diagram

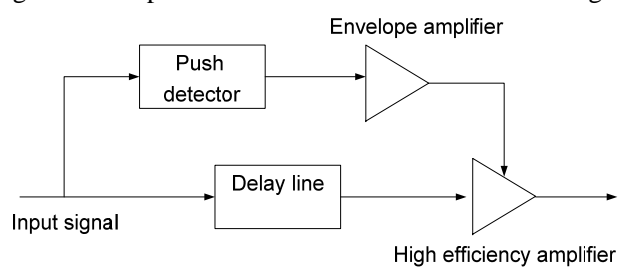

Fig. 3. hybrid eer block diagram

\section{ENVELOPE AMPLIFIER}

Dynamic supply control in EER and ET can enhance the RF PA drain/collector average efficiency, but the total system efficiency is determined by the product of the envelope amplifier efficiency and the RF transistor drain/collector efficiency. As a result, a high-efficiency envelope amplifier design is vital to the EER/ET system. The high efficiency envelope amplifier is usually realized by a DC/DC converter, and the switching frequency is required to be several times the signal bandwidth [16]. For narrow bandwidth applications, most high efficiency switching mode DC/DC converters are realized by traditional delta modulation [17] or pulse width modulation (PWM) [18] modulators. On the other hand, the high switching frequency will bring in a considerable switching loss for a wideband signal. For instance, in ET PA for a WLAN OFDM signal for low EVM, a $20 \mathrm{MHz}$ envelope bandwidth is necessary. Hence, the switching frequency of the traditional DC/DC converter will be no less than $100 \mathrm{MHz}$, which introduces an important switching loss [3]. Recently, a switching circuit is proposed for envelope amplifier, which has capability of having high bandwidth and efficiency. Mathematical behavioral model of this envelope amplifier is shown in Fig. 4, which consists of a voltage source and a current source. Although the current source has high efficiency, the voltage source has low efficiency. Therefore, we like to get most of the output current from the current source. To control the current of the voltage source, we use a hysteretic current feedback control to realize the soft power division between switch stage and linear stage amplification.

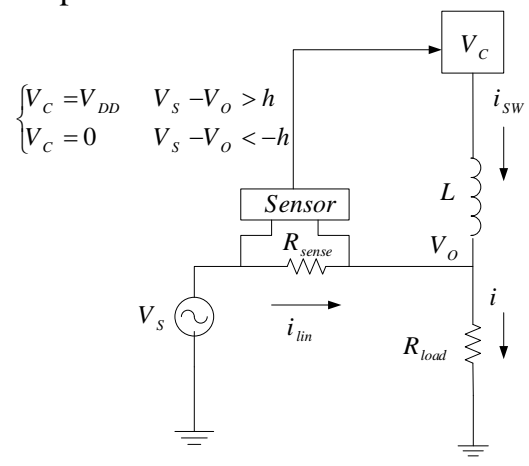

Fig. 4. Mathematical behavioral model of the envelope amplifier

The load voltage is controlled by the linear voltage source, and the load current is a mixture of the linear stage current and the switch stage current. Envelope amplifier block diagram is shown in Fig. 5, which consists of an OPAMP to realize the voltage source, and a MOSFET to realize the current source.

\section{SimULATION}

\section{A. RF Amplifier Design}

Si-LDMOS has been a popular device choice for base-station high-power amplifiers, since LDMOS technology can provide reliable and cost effective solutions [19]. Envelope tracking techniques, in which a wideband envelope amplifier makes variable supply biasing to the RF stage, have established excellent performance using a variety of device technologies including Si LDMOS [20] and GaN FETs [21].

Nonetheless the RF PA should have suitable characteristics to be appropriate for the envelope tracking operation and achieve the optimal performance such as low deviation of the output capacitance, since a large variation of the voltage dependent output capacitance will corrupt the average efficiency as the optimum impedance matching for the output of the PA changes with the supply voltage [18]. Besides, extra nonlinearity like AM-PM distortion from the nonlinear capacitance and AM-AM distortion from the envelope amplifier, and memory effects due to the limited bandwidth of the RF PA and the envelope amplifier is produced by the dynamic supply biasing. However, we can use digital pre-distortion (DPD) techniques to correct the nonlinearity of the dynamically biased amplifier [7].

The amplifier is designed and implemented using the MRF6S27015N MOTOROLA transistor in LDMOS technology by ADS2008 software. The design of the amplifier is at the central frequency of $2.1 \mathrm{GHz}$ and the bandwidth of the RF amplifier is $200 \mathrm{MHz}$, which is high with respect to the other works.

Our aim in the design of the power amplifier is to increase the efficiency. So, we optimized the amplifier to reach our goal. We changed Vgs ; Vds, width and length of the matching transmission lines to have a good efficiency. 
We know that increasing the output power of the transistor leads to increase in PAE. It means that we have the best efficiency in the peak power. However, our input signal has a high peak to average ratio and the probability of the peak power is very low. Consequently, it is wise to design the matching for the case that happens most of the time, which is mean power. Therefore, because Vds changes with respect to the input power, we optimize the power amplifier for the Vds related to the mean power.

Gain, power added efficiency and Output power of the RF power amplifier in the frequency band is shown in Fig. 7, Fig. 8 and Fig. 9 respectively.

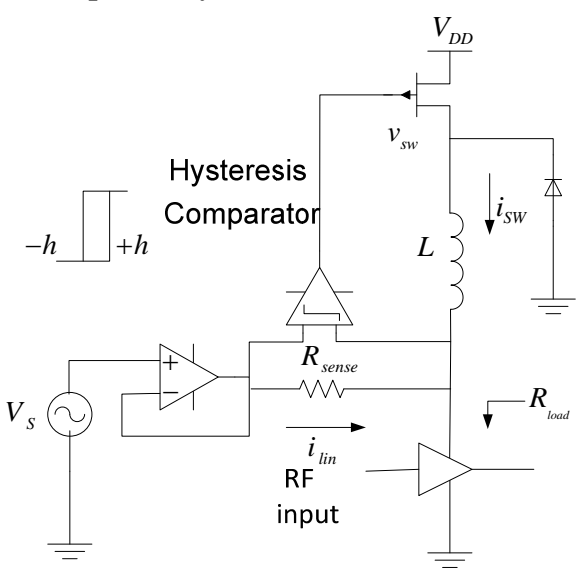

Fig. 5. Envelope amplifier block diagram

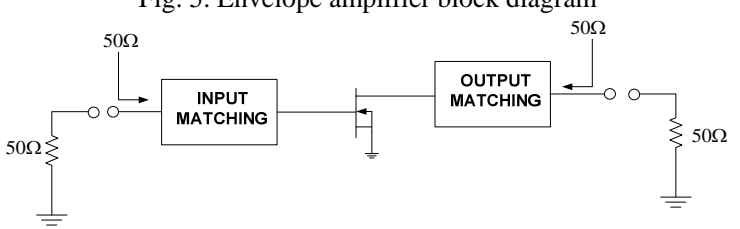

Fig. 6. RF amplifier block diagram

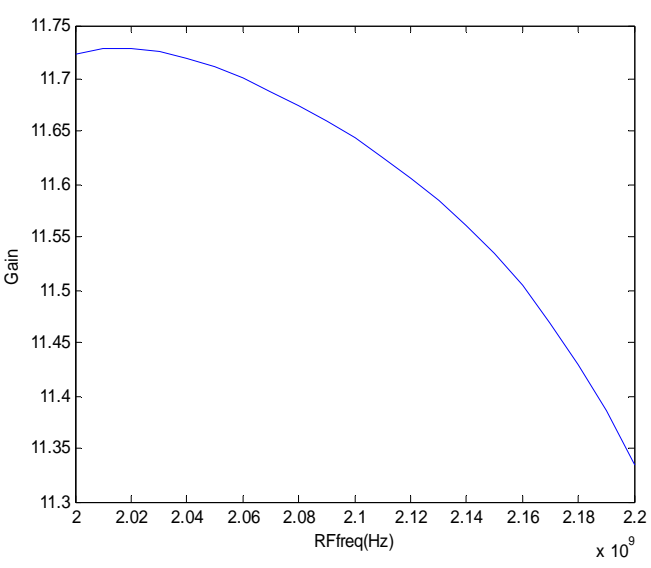

Fig. 7. Gain versus frequency by Vds $=8(\mathrm{v})$

\section{B. Changing Vds}

In next step, we changed Vds of the power amplifier from $8 \mathrm{v}$ up to $28 \mathrm{v}$ by $4 \mathrm{v}$ to show the possibility of increase in PAE by envelope tracking. Gain, power added efficiency and Output power of the RF power versus input power at center frequency of $2.1 \mathrm{GHz}$ by changing $\mathrm{Vds}$ from $8 \mathrm{v}$ up to $28 \mathrm{v}$ by $4 \mathrm{v}$ is shown in Fig. 10, Fig. 11 and Fig. 12 respectively. When the input power is $10 \mathrm{dBm}$ and $\mathrm{Vds}$ is $8 \mathrm{v}$, this simulation shows about $17 \%$ of more PAE than the condition of $\mathrm{Vds}=28 \mathrm{v}$. Fig. 12 shows that we can increase P1dB of the transistor when the input power is large by applying ET.

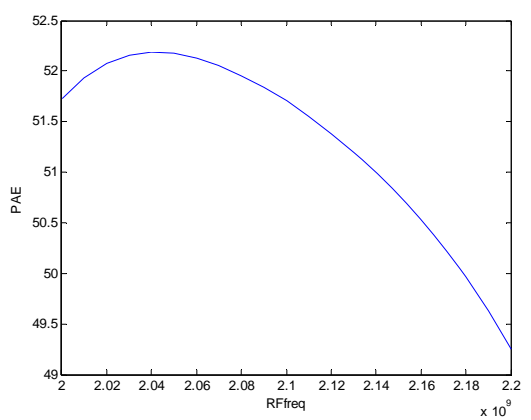

Fig. 8. Power Added Efficiency versus frequency

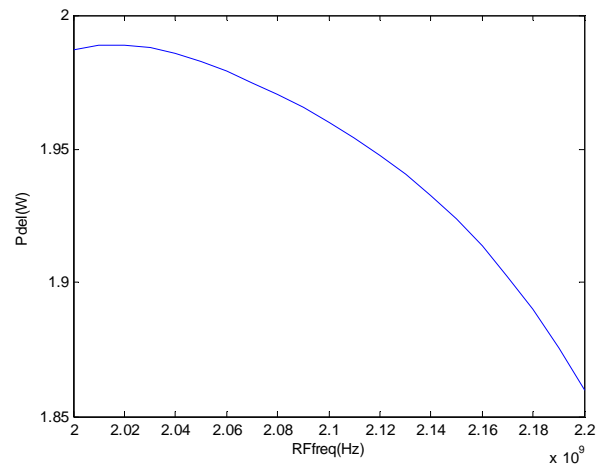

Fig. 9. Output power versus frequency

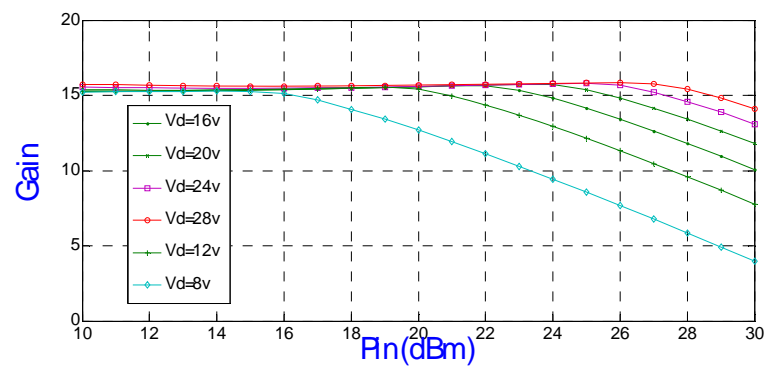

Fig. 10. Gain versus input power at center frequency by changing Vds

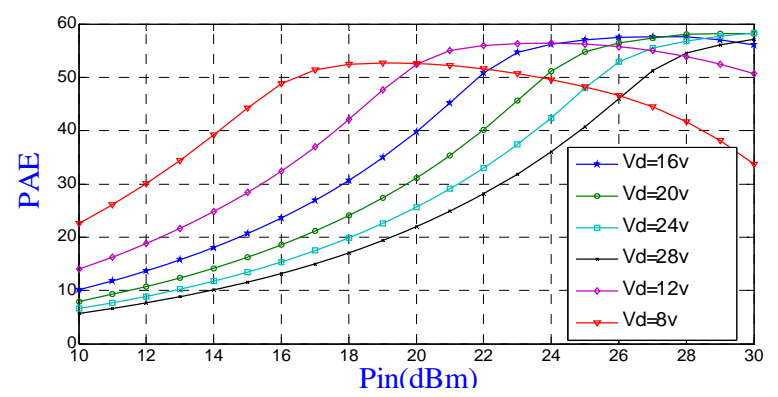

Fig. 11. PAE versus input power at center frequency by changing Vds

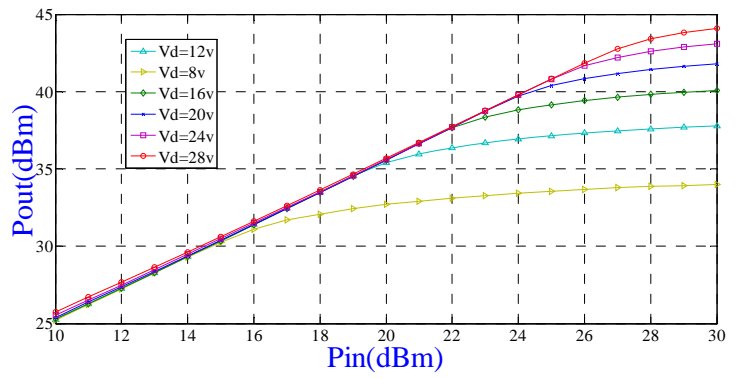

Fig. 12. Output power versus input power at center frequency by changing Vds 


\section{Applying ET}

When $\mathrm{Vds}$ is $8 \mathrm{v}$ the PAE decreases in the peak power, and when $\mathrm{Vds}$ is $28 \mathrm{v}$ the PAE is low in weak power. To overcome this problem, we can apply envelope tracking to our amplifier. By changing the Vds with respect to the input power, we can see that the PAE remains above $50 \%$ for a wide range of input power. Fig. 13 and Fig. 14 show PAE and Gain versus input power at center frequency by applying envelope tracking respectively.

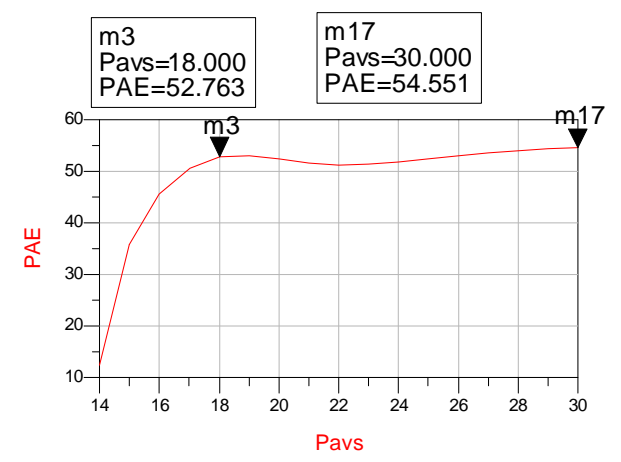

Fig. 13. PAE versus input power at center frequency by applying envelope tracking

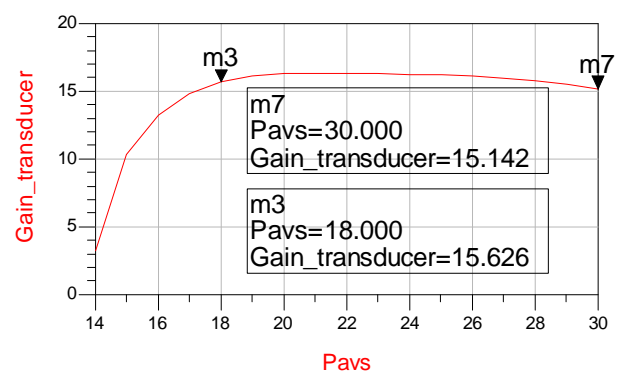

Fig. 14. Gain versus input power at center frequency by applying envelope tracking

\section{CONCLUSION}

In this research we have described EER, ET and HEER methods. HEER amplifier for frequency range of 2 to 2.2 $\mathrm{GHz}$ for use in transmitters to produce 2 watts of power is introduced. The amplifier is designed and implemented using the MRF6S27015N MOTOROLA transistor in LDMOS technology. We have shown that the PAE remains above $50 \%$ for a wide range of input power. We have $200 \mathrm{MHz}$ of bandwidth.

\section{ACKNOWLEDGMENT}

We thanks microwave/mm-wave and wireless communication research laboratory of Amirkabir University.

\section{REFERENCES}

[1] G. D. Gordon and W. L. Morgan, Principles of Communications Satellites, New York: Wiley, 1993.

[2] Y. Woo, J. Yi, Y. Yang, and B. Kim, "SDR transmitter based on LINCamplifier with bias control,” IEEE MTT-S Int. Microwave Sympo. Dig, vol. 3, pp. 1703-1706, Jun. 2003.
[3] J. Choi, J. Yim, J. Yang, J. Kim, J. Cha, and B. Kim, “A Delta-Sigma-Digitized RF transmitter,” IEEE MTT-S Int. Microwave Sympo. Dig, 2007.

[4] S. C. Cripps, "RF Power Amplifiers for Wireless Communications," Norwood, MA: Artech House, 2006.

[5] F. H. Raab, P. Asbeck, S. Cripps, P. B. Kenington, Z. B. Popovic, N. Pothecary, J. F. Sevic, and N. O. Sokal, "Power amplifiers and transmittersfor RF and microwave," IEEE Trans. Microwave Theory and Tech, vol. 50, no. 3, pp. 814-826, Mar. 2002

[6] N. Schlumpf, M. Declercq, and C. Dehollain, “A fast modulator for dynamic supply linear RF power amplifier,” IEEE J. Solid-State Circuits, vol. 39, pp. 1015-1025, July 2004.

[7] F. Wang, A. Ojo, D. Kimball, P. Asbeck, and L. Larson, "Envelope tracking power amplifier with pre-distortion for WLAN 802.11g," IEEE MTT-S Int. Microwave Symp. Dig, 2004, pp. 1543-1546.

[8] F. Wang, A. Yang, D. Kimball, L. Larson, and P. Asbeck, "Design of wide-bandwidth envelope-tracking power amplifiers for OFDM applications," IEEE Trans. Microwave Theory Tech, vol. 53, pp. 1244-1255, April 2005.

[9] G. Hanington, P. Chen, P. M. Asbeck, and L. E. Larson, "High efficiency power amplifier using dynamic power-supply voltage for CDMA applications,” IEEE Trans. Microwave Theory Tech, vol. 47, pp. 1471-1476, Aug. 1999.

[10] J. Staudinger, B. Gilsdorf, D. Newman, G. Norris, G. Sadowniczak, R. Sherman, and T. Quach, "High efficiency CDMA power amplifier using dynamic envelope tracking technique," IEEE MTT-S Int. Microwave Symp. Dig, 2000, pp. 873-976.

[11] B. Sahu, G. A. R. Mora, “A High-efficiency linear RF power amplifier with power-tracking dynamically adaptive buck-boost supply,” IEEE Trans. Microwave Theory Tech, vol. 52, pp. 112-120, Jan. 2004.

[12] A. Khanifar, N. Maslennikov, R. Modina, and M. Gurvich, "Enhancement of power amplifier efficiency through dynamic bias switching," in IEEE MTT-S Int. Microwave Symp. Dig, 2004, pp. 2047-2050.

[13] J. Deng, P. Gudem, L. Larson, and D. Kimball, “A SiGe PA with dual dynamic bias control and memoryless digital predistortion for WCDMA handset applications," in IEEE Radio Frequency IC Symp. Dig, 2005.

[14] L. Kahn, "Single-sideband transmission by envelope elimination and restoration,” in Proc. IRE, July 1952, pp. 803-806.

[15] F. Wang, D. Kimball, J. Popp, A. Yang, D. Y. C. Lie, P. Asbeck, and L. E. Larson, "Wideband envelope elimination and restoration power amplifier with high efficiency wideband envelope amplifier for WLAN 802.11g applications,” IEEE MTT-S Int. Microwave Symp. Dig, Long Beach, CA, June 12-17, 2005.

[16] F. H. Raab, "Intermodulation distortion in Kahn-technique transmitters," IEEE Trans. Microwave Theory Tech, vol. 44, pp. 2273-2278, Dec. 1996.

[17] D. R. Anderson and W. H. Cantrell, "High efficiency high level modulator for use in dynamic envelope tracking CDMA RF power amplifiers," IEEE MTT-S Int. Microwave Symp. Dig, 2001, pp. 1509-1512.

[18] B. Sahu and G. A. R. Mora, “A low voltage, dynamic, noninverting, synchronous buck-boost converter for portable applications,” IEEE J. Solid-State Circuits, vol. 19, pp. 443-452, March 2004.

[19] W. Burger, H. Brech, D. Burdeaux, C. Dragon, G. Formicone, M. Honan, B. Pryor, and X. Ren, "RF-LDMOS: A device technology for high power RF infrastructure applications,” in Proc. IEEE Compound Semicond. Integr. Circuit Symp, Oct. 2004, pp. 189-192.

[20] C. Hsia, D. Kimball, P. Draxler, J. J. Yan, J. Kinney, E. Toulouse, J. Wood, and P. M. Asbeck, "High efficiency envelope tracking overdrivenclass-A LDMOS power amplifier for base station applications," IEEE Topical Symp. Power Amplifiers for Wireless Commun, Orlando, FL, Jan. 2008.

[21] D. F. Kimball, J. Jeong, C. Hsia, P. Draxler, S. Lanfranco, W. Nagy, K. Linthicum, L. E. Larson, and P. M. Asbeck, "High efficiency envelope tracking W-CDMA base-station amplifier using GaN HFETs,” IEEE Trans. Microw. Theory Tech, vol. 54, no. 11, pp. 3848-3856, Nov. 2006. 